day (P) 7). Both groups received iNO (5 ppm) or air from E21 to P7. Animals were evaluated at P3, P10 and P21 using immunohistochemistry, cognitive functions and mass spectrometry imaging.

iNO significantly attenuated the severity of hyperoxia-induced WMD induced in neonatal rats. Specifically, iNO decreased white matter inflammation, cell death, and enhanced the density of developing oligodendrocytes and oligodendroglial maturation. Furthermore, iNO triggered an early upregulation of P27kip1 and brain-derived growth factor (BDNF). Whereas hyperoxia disrupted early associative abilities, iNO treatment maintained learning scores to a level similar to that of control pups. In contrast to its marked neuroprotective effects, iNO induced only small and transient improvements of CLD.

These findings suggest that iNO exposure at low doses is specifically neuroprotective in an animal model combining simultaneously injuries of the developing lung and brain that mimicked CLD and $\mathrm{WMD}$ in preterm infants.

\section{THE SIGMA-1 RECEPTOR AGONIST PRE-084 ATTENUATES INFLAMMATION-SENSITIZED NMDAR-MEDIATED EXCITOTOXIC BRAIN INJURY IN NEWBORN MICE}

doi:10.1136/archdischild-2012-302724.0009

${ }^{1} \mathrm{~A}$ Posod, 'K Neumayer, 'V Neubauer, ${ }^{1,2} \mathrm{M}$ Keller, ${ }^{1} \mathrm{~K}$ Wegleiter, 'M Urbanek, 'U KiechlKohlendorfer, 'E Griesmaier. 'Department of Paediatrics IV, Division of Neonatology, Innsbruck Medical University, Innsbruck, Austria; 'Department of Paediatrics, Children's Hospital Passau, Passau, Germany

Excitotoxicity and inflammation play crucial roles in the etiopathogenesis of perinatal brain injury. We have shown that the sigma-1 receptor agonist 2-(4-morpholinethyl) 1-phenylcyclohexanecarboxylate (PRE-084) protects against $\mathrm{N}$-methyl-d-aspartate (NMDA) receptor-mediated excitotoxic brain injury. In models of adult central nervous system pathology, PRE-084 has demonstrated potent anti-inflammatory properties, which makes it a promising candidate for countering inflammation-enhanced perinatal brain injury.

In the present study we evaluated the effect of PRE-084 in a neonatal mouse model of inflammation-sensitized excitotoxic brain injury.

From postnatal days 1 to 4, pups were pre-sensitized by intraperitoneal injections of IL-1beta (10ng). Two hours after the last IL-1beta dose, pups received an intracranial ibotenate injection, 1 hour after the insult they were randomly treated with i) $0.1 \mu \mathrm{g} / \mathrm{g}$ bodyweight PRE-084 or ii) vehicle.

Administration of PRE-084 resulted in a significant decrease in cortical grey (mean length of the lesion: vehicle $780.00 \mu \mathrm{m} \pm 495.35$ vs. PRE-084 433.33 $\mu \mathrm{m} \pm 116.51 ; \mathrm{n}=8-9 ; \mathrm{p}<0.05)$ and adjacent white matter damage (mean length of the lesion: vehicle $767.50 \mu \mathrm{m} \pm$ 489.07 vs. PRE-084 391.11 $\mu \mathrm{m} \pm 126.14 ; \mathrm{n}=8-9 ; \mathrm{p}<0.05)$. No sexspecific differences in lesion size were detected ( $n=3-6, p>0.05$ ). PRE-084 treatment significantly reduced the number of isolectin B4-positive activated microglial cells in perilesional white matter (mean number of isolectin B4-positive activated microglia vehicle $36.40 \pm 6.96$ vs. PRE-084 19.93 $\pm 11.99 ; n=5 ; p<0.05)$.

We are the first to report that PRE-084 reduces inflammationsensitized NMDAR-mediated excitotoxic perinatal brain damage. Since sigma-1 receptor agonists are investigated in clinical trials in adult neurological diseases, they might be considered a promising therapeutic option also in perinatal brain injury.

\section{MTOR ACTIVATES HYPOXIA-INDUCIBLE FACTOR-1 $\alpha$ AND INHIBITS NEURONAL APOPTOSIS IN THE DEVELOPING RAT BRAIN DURING THE EARLY PHASE AFTER HYPOXIA- ISCHEMIA}

doi:10.1136/archdischild-2012-302724.0010
${ }^{1} \mathrm{H}$ Chen, ${ }^{1} \mathrm{~T}$ Xiong, ${ }^{1} \mathrm{Y} \mathrm{Ou},{ }^{1} \mathrm{~F}$ Zhao, ${ }^{2} \mathrm{D}$ Ferriero, ${ }^{1,2} \mathrm{D} \mathrm{Mu}$. ' $\mathrm{Cepartment}$ of Pediatrics, West China Second University Hospital, Sichuan University, Chengdu, China; ${ }^{2}$ Department of Neurology, University of California, San Francisco, CA, USA

The mammalian target of rapamycin (mTOR) exerts neuroprotective effects under hypoxic or ischemic conditions. To explore whether mTOR participates in neuroprotective signaling through regulation of hypoxia-inducible factor- $1 \alpha$ (HIF-1 $\alpha$ ), vascular endothelial growth factor (VEGF) and neuronal apoptosis in developing rat brain with hypoxia-ischemia (HI), we operated on postnatal day 10 rats by ligating the common carotid artery followed by exposure to systemic hypoxia. Brains were collected at various intervals to detect the expression of mTOR, phosphorylated mTOR (p-mTOR), HIF-1 $\alpha$, VEGF and cleaved caspase 3 (CC3), using immunohistochemistry and Western blot analysis. We also used terminal deoxynucleotidyl transferase-mediated dUTP-nick end labeling (TUNEL) to detect neuronal apoptosis. The p-mTOR protein expression increased at $2 \mathrm{~h}$ after $\mathrm{HI}$, peaked at $8 \mathrm{~h}$, lasted $24 \mathrm{~h}$, and then dropped to the basal level. Also, the expression of HIF-1 $\alpha$ and VEGF was significantly enhanced and peaked at $8 \mathrm{~h}$ after HI. Up-regulated expression of CC3 was observed at $2 \mathrm{~h}$, peaked at $24 \mathrm{~h}$, and lasted $72 \mathrm{~h}$ after $\mathrm{HI}$. Increased neuronal apoptosis is associated with reduced HIF- $1 \alpha$ and VEGF expression. Furthermore, pretreatment with rapamycin, a mTOR specific inhibitor, significantly inhibited HIF- $1 \alpha$ and VEGF protein after HI. The expression of CC3 and the number of TUNEL-positive cells were up-regulated at $8 \mathrm{~h}$ and down-regulated at $24 \mathrm{~h}$ after $\mathrm{HI}$ in the rapamycin-treated group. Our findings suggest that $\mathrm{mTOR}$ may participate in the regulation of HIF-1 $\alpha$, VEGF and neuronal apoptosis, serving neuroprotective functions after $\mathrm{HI}$ in developing rat brain.

\section{FLUID THERAPY SHOULD BE GUIDED BY FLUID RESPONSIVENESS}

doi:10.1136/archdischild-2012-302724.0011

J López-Herce, J Urbano. Pediatric Intensive Care Department, Hospital General Universitario Gregorio Marañón, Madrid, Spain

Background To predict fluid response is very Important because a little or excessive expansion may alter the prognosis of the child in shock

Methods We review experimental and clinical articles in adult and children about parameters that could predict fluid responsiveness in shock. We also analyze our experimental data in pediatric experimental model of hemorrhagic shock.

Results The most used parameters to try to predict hemodynamic response to fluids are: static pressure parameters as central venous pressure (CVP); volume as global end diastolic ventricular index (GEDVI) or stroke volume index (SVI); dynamic parameters, as pulse pressure variation (PPV) and systolic volume variation (SVV), and the response to a maneuver that increases blood volume without expanding the patient (leg raises). Several studies in adults suggest that hemodynamic volume parameters (SVI or GEDVI) predict better the response to fluids than pressure parameters (PVC); that dynamic parameters (PPV and SVV) predict better the response to fluids that static parameters; and that maneuver leg raises maneuver is the best predictive parameter. However, the results of other studies are contradictory. In children there are few studies and there is no evidence that dynamic parameters are better predictors than static volume parameters. Our experimental studies confirm these findings. Preliminary data suggest that leg raise maneuver has not good predictive power in children.

Conclusion at this time fluid therapy in children with shock should be guided by fluid responsiveness. Macrohemodynamic, microhemodynamic and tissue parameters should be used to control the response to fluid therapy. 